\title{
Interference Reduction in Glucose Detection by Redox Potential Tuning: New Glucose Meter Development
}

\author{
Seong Je Cho,* Chul-Ho Cho,* Kwang Bok KIm,* Min-Hyoung LeE,* Jae Hong KIm,* Suho LeE,* \\ Jaegeol CHO,* Suntae Jung,* Dong-Min KIM,** and Yoon-Bo SHIM**ं \\ *DMC R\&D center Samsung Electronics, 129, Samsung-ro, Yeongtong-gu, Suwon-si, Gyeonggi-do 443-742, \\ South Korea \\ **Department of Chemistry and Institute of Biophysio Sensor Technology (IBST), Pusan National University, \\ Busan 609-735, South Korea
}

\begin{abstract}
A new glucose meter was developed employing a novel disposable glucose sensor strip comprising a nicotinamide adenine dinucleotide-glucose dehydrogenase (NAD-GDH) and a mixture of Fe compounds as a mediator. An iron complex, 5-(2,5-di(thiophen-2-yl)-1H-pyrrol-1-yl)-1,10-phenanthroline iron(III) chloride (Fe-PhenTPy), was synthesized as a new mediator for the NAD-GDH system. Due to the high oxidation potential of the mediator, the detection potential was tuned to be more closely fitted toward the enzyme reaction potential, less than $400 \mathrm{mV}$ (vs. $\mathrm{Ag} / \mathrm{AgCl}$ ), by mixing with an additional iron mediator. The impedance spectrometry for the enzyme sensor containing the mixed mediators showed an enhanced charge transfer property. In addition, a new cartridge-type glucose meter was manufactured using effective aligned-electrodes, which showed an enhanced response compared with conventional electrode alignment. The proposed glucose sensor resulted in a wide dynamic range in the concentration range of $30-500 \mathrm{mg} \mathrm{dL}^{-1}$ with a reduced interference effect and a good sensitivity of $0.57 \mu \mathrm{A} \mathrm{mM}{ }^{-1}$.
\end{abstract}

Keywords Glucose sensor, mixed Fe-complex mediator, NAD-GDH, potential tuning

(Received January 28, 2015; Accepted March 2, 2015; Published July 10, 2015)

\section{Introduction}

Numerous clinical studies for diabetes have demonstrated that intensive blood glucose control is essential to patients for preventing the risk of serious complications. ${ }^{1}$ The Diabetes Control Complications Trial (DCCT) reported that active blood glucose control has reduced the risk of eye, kidney, and nerve diseases by 76,50 , and $60 \%$, respectively. ${ }^{2}$ With an increase in the number of diabetes patients, point-of-care technique (POCT) is an important constituent of modern therapy and methods to enable the maintenance of a constant glucose level. Thus, numerous researchers have developed glucose biosensors to precisely monitor the blood glucose level. In addition, there are several clinical methods used to monitor the glucose concentration, such as colorimetry, fluorescent spectroscopy, conductometry, and electrochemical method. ${ }^{3-6}$ Nowadays, electrochemical techniques are mostly used to monitor glucose due to the high selectivity, sensitivity, low cost, fast response time, and high reproducibility.

The majority of commercialized electrochemical sensors are based on enzymatic reactions, which are constructed with an artificial electron-transfer mediator between the redox center of the enzyme and the electrode surface. Two kinds of enzymes for commercialized glucose sensors are glucose oxidase (GOx) and glucose dehydrogenase (GDH). Of these, GOx have been

† To whom correspondence should be addressed.

E-mail: ybshim@pusan.ac.kr considered as a standard enzyme for the glucose sensor, so far. Since GOx easily catalyzes the oxidation of $\beta$-D-glucose to glucono-1,5-lactone with oxygen molecule as the electron acceptor, the GOx modified electrode can determine the levels of glucose by monitoring the current involved in the process. However, the enzymatic reaction of GOx is dependent on the dissolved oxygen in the blood, which is one of the disadvantages. To overcome the oxygen dependence problem of GOx, GDHbased glucose sensor systems have been studied recently to commercialize them.

There are many available coenzymes in GDH-based systems. Notable examples of these include nicotinamide adenine dinucleotide (NAD), flavin adenine dinucleotide (FAD), and pyrroloquinoline quinone (PQQ). ${ }^{7-9}$ Of these, PQQ-GDH shows an indiscriminate response to maltose, galactose and xylose, thus misinterpreting those sugars as glucose. ${ }^{10}$ The US Food and Drug Administration (FDA) has identified some products containing or metabolized into maltose, galactose, or xylose, as potential interfering products with PQQ-GDH glucose sensors. FAD-GDH also shows an indiscriminate response to xylose. On the other hand, NAD-GDH exhibits to be highly selective to $\beta$-D-glucose over other monosaccharides. Additionally, coenzymes for electron transfer involved in the enzyme reaction were extensively studied for shuttling electrons between the redox center of the enzyme and the electrode rapidly. Most mediators are commonly used for the FAD-GOx or FAD-GDH system. However, only a few adequate mediators for NAD$\mathrm{GDH}$, such as quinone, quinoid dyes, and 1,10-pheanathroline quinone, were employed for glucose sensor strips. ${ }^{11}$ Thus, we 
recently reported on a novel electron-transfer mediator (Fe-PhenTPy) in NAD-GDH based glucose sensors, with a bisthiophene-pyrrole backbone bearing an iron-phenanthroline complex. ${ }^{12}$ However, the drawback of this NAD-GDH system is the high working potential, which may induce interferences from easily oxidizing species present in blood, such as AA (ascorbic acid), AP (acetaminophen), and UA (uric acid) without any protecting polymer membrane. Thus, the oxidation potential of the mediator needs to be tuned a lower potential than that of other electrochemically active interference species so as to avoid their interferences. In addition, it is necessary to control the polarity of the mediator to enhance the absorptivity of mediator on the carbon electrode surface, which is also affects the sensor performance.

To commercialize an improved NAD-GDH-based glucose sensor, using of a mediator having a low oxidation potential so as to avoid interferences coexisting in a blood sample is a lasting hurdle to be overcome. Thus, the present study examined the tuning of the oxidation potential of the prepared mediator by mixing with an additional mediator and by regulating the absorptivity through polarity control. For this purpose, we simply blended two compounds (Fe-PhenTPy and potassium hexacyano ferrate $\left.\left(\mathrm{Fe}(\mathrm{CN})_{6}{ }^{3-}\right)\right)$ in different ratios and then dissolved them in a mixing solvent. The proposed glucose sensor was evaluated regarding various interfering species in human blood according to International Organization for Standardization (ISO) 15197. In addition, the present study demonstrates a new glucose meter system equipped with the NAD-GDH glucose sensor containing the mixed mediator for detection potential tuning. We also aligned the structure of three electrodes in the sensor strip so as to reveal the highest responsibility.

\section{Experimental}

\section{Materials}

Tetrabutylammonium perchlorate (TBAP, electrochemical grade) was purchased from Fluka (USA). It was purified according to a general method, and dried under a vacuum at $1.33 \times 10^{-3} \mathrm{~Pa}$. $\mathrm{NAD}^{+}$dependent glucose dehydrogenase was purchased from Toyobo Co. (Japan). Dimethyl sulfoxide (DMSO, 99.9\%, anhydrous, sealed under $\mathrm{N}_{2}$ gas), nicotinamide adenine dinucleotide oxidize form $\left(\mathrm{NAD}^{+}\right)$, glucose, fructose, lactose, mannose, xylose, ascorbic acid, acetaminophen were purchased from Sigma Co. (USA). All of the aqueous solutions were prepared in doubly distilled water, which was obtained from a Milli-Q water-purifying system $(18 \mathrm{M} \Omega \mathrm{cm})$.

\section{Instruments}

Cyclic voltammetry for mediators was performed in a threeelectrode system, where glassy carbon, $\mathrm{Ag} / \mathrm{AgCl}$ (in saturated $\mathrm{KCl}$ ), and $\mathrm{Pt}$ wire were used as the working, reference, and counter electrode, respectively. Screen printed sensor strips were used for fabricating an NAD-GDH based-glucose sensor. The working ( area $=0.02 \mathrm{~cm}^{2}$ ), pseudo reference, and counter electrode were all made of carbon. The potential of carbon pseudo reference electrode was constant in a blood sample, which shows a non-polarized electrode behavior. A commercial carbon ink (Dupont Inc.) was used in the screen printing process. The SPCEs were printed on polystyrene-based film from a screen printer (BANDO industrial, Korea). Cyclic voltammograms (CVs), and amperograms were recorded using a potentiostat/galvanostat, Kosentech Model PT-1 (South Korea). Electrochemical impedance spectroscopy was performed using an EG\&G Princeton Applied Research PARSTAT 2263. The frequency was scanned from $100 \mathrm{kHz}$ to $1 \mathrm{~Hz}$ at the open-circuit voltage, acquiring five points per decade.

\section{Preparation of mediators}

We synthesized a new novel mediator for the NAD-GDH sensor, Fe-complex (Fe-PhenTPy; 5-(2,5-di(thiophen-2-yl)-1Hpyrrol-1-yl)-1,10-phenanthroline iron(III) chloride). ${ }^{12}$ The detail synthesis procedure is as follows: 1,10-phenanthrolin-5-amine (590 mg; $3.03 \mathrm{mM})$, 1,4-di(2-thienyl)butane-1,4-dione (765 mg; $3.05 \mathrm{mM}$ ), and PTSA (74 mg; $0.43 \mathrm{mM}$ ) were dissolved in dry toluene $(200 \mathrm{~mL})$ in an RB flask fitted with a Soxhlet extractor filled with $3 \AA$ molecular sieves and a condenser. The solution was refluxed for $48 \mathrm{~h}$, and the reaction was monitored by TLC using $100 \% \mathrm{CH}_{2} \mathrm{Cl}_{2}$ as an eluent. After the allotted time, the mixture was cooled to room temperature and the toluene was removed in a rotary evaporator. The residue was re-dissolved in a minimum volume of $\mathrm{CH}_{2} \mathrm{Cl}_{2}$ and applied on a silica column. Elution with $100 \% \mathrm{CH}_{2} \mathrm{Cl}_{2}$ was afforded rapidly off the column to get a pure product, 5-(2,5-di-(thiophen-2-yl)-1H-pyrrol-1-yl)1,10-phenanthroline (PhenTPy) $1.05 \mathrm{~g}$ (85\%). A solution of $\mathrm{FeCl}_{3}(400 \mathrm{mg} ; 2.50 \mathrm{mM})$ in absolute ethanol $(20 \mathrm{~mL})$ was added with stirring to a solution of PhenTPy $(1.0 \mathrm{~g} ; 2.40 \mathrm{mM})$ in absolute ethanol $(30 \mathrm{~mL})$. The formation of deep-red precipitates was immediately formed and the reaction mixture was stirred at the room temperature for about $1 \mathrm{~h}$. The supernatant was filtered off and the precipitates were washed with ethanol and dried under a vacuum. Yield $1.12 \mathrm{~g}(82.5 \%)$. The purity of Fe-PhenTPy was confirmed (>99\%) by ${ }^{1} \mathrm{H}-\mathrm{NMR}$ and mass spectroscopy. (Data not shown)

\section{Fabrication of sensor probe}

Glucose sensors based on NAD-GDH were fabricated using $\mathrm{Fe}(\mathrm{CN})_{6}{ }^{3-}$, Fe-PhenTPy and enzyme (NAD-GDH). Firstly, $2.0 \mathrm{mM}$ Fe-PhenTPy was dissolved in $0.2 \mathrm{~mL}$ DMSO, and then added with $0.8 \mathrm{~mL}$ EtOH $+\mathrm{H}_{2} \mathrm{O}(1: 3, \mathrm{v} / \mathrm{v})$. Then, $1.5 \mu \mathrm{L}$ of Fe-complex was dropped onto the SPCE and dried at $35^{\circ} \mathrm{C}$ for 5 min. Subsequently, $\mathrm{Fe}(\mathrm{CN})_{6}{ }^{3-}$ in a PVA solution $(2 \mathrm{wt} \%)$ of $1.5 \mu \mathrm{L}$ was drop onto the Fe-PhenTPy modified SPCE and dried at $35^{\circ} \mathrm{C}$ for $5 \mathrm{~min}$. An enzyme solution was prepared by

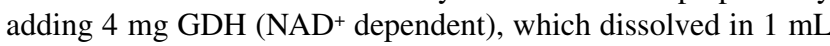
of distilled water, and $5 \mathrm{mM} \mathrm{NAD}^{+}$was added into the GDH solution. Finally, $3 \mu \mathrm{L}$ of NAD-GDH was dropped onto the $\mathrm{Fe}(\mathrm{CN})_{6}{ }^{3-} / \mathrm{Fe}-\mathrm{PhenTPy} / \mathrm{SPCE}$ and dried at $35^{\circ} \mathrm{C}$ for $5 \mathrm{~min}$.

\section{Results and Discussion}

Synthesis and voltammetric behaviors of mediators in DMSO

A mediator (Fe-PhenTPy) was synthesized from $\mathrm{FeCl}_{3}$, and ligand (PhenTPy) through a Paal-Knorr pyrrole condensation reaction in the presence of a PTSA catalyst in dry toluene. The phenanthroline iron moiety was functioned as a mediator for the NAD(P)-GDH enzyme system with good selectivity. Fecomplex bearing a dithiophen pyrrole group was adsorbed on the carbon electrode by the $\pi-\pi$ interaction due to its delocalized aromatic rings. The voltammetric characterization of the FePhenTPy was performed to evaluate its redox property in a DMSO solution containing $0.1 \mathrm{M}$ TBAP. Figure 1 shows the $\mathrm{CVs}$ recorded for the mediator from -1.0 to $0.7 \mathrm{~V}$ (vs. $\mathrm{Ag} / \mathrm{AgCl})$ at a scan rate of $50 \mathrm{mV} \mathrm{s}^{-1}$, where the redox potentials of Fe-PhenTPy were $310 / 210 \mathrm{mV}$ in DMSO (Fig. 1(A)). This potential is quite positive, which derived the oxidation reactions of interferences, such as ascorbic acid, acetaminophen, 

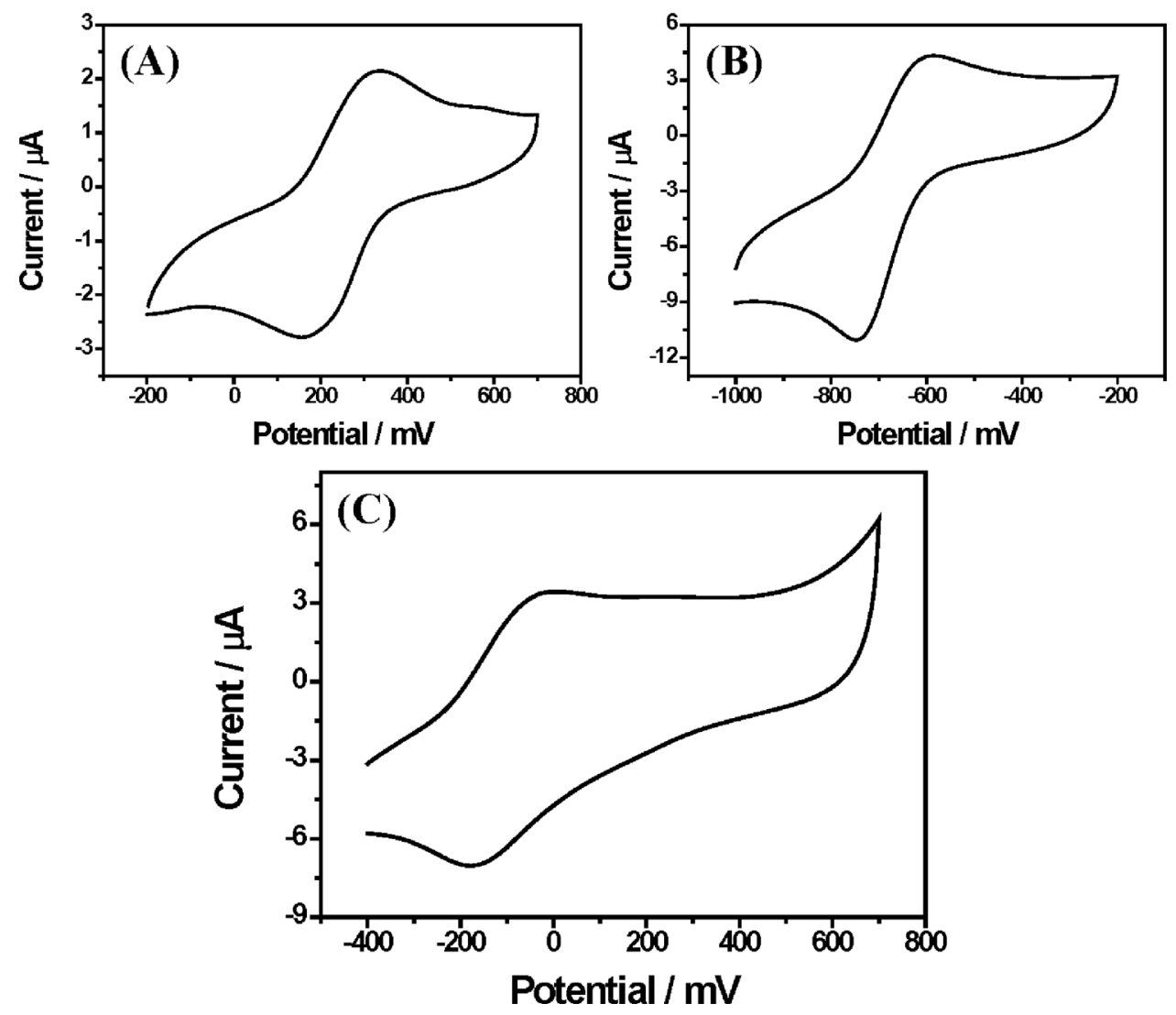

Fig. 1 CVs recorded for the (A) Fe-PhenTPy, (B) $\mathrm{Fe}(\mathrm{CN})_{6}{ }^{3-}$, and (C) Fe-PhenTPy/ $\mathrm{Fe}(\mathrm{CN})_{6}{ }^{3-}$ in the DMSO containg $0.1 \mathrm{M}$ TBAP.

dopamine, etc. Thus, we tried to reduce the oxidation potential of $\mathrm{Fe}$-PhenTPy by mixing with $\mathrm{Fe}(\mathrm{CN})_{6}{ }^{3-}$. As shown in Fig. 1(B), the redox potential of $\mathrm{Fe}(\mathrm{CN})_{6}{ }^{3-}$ was $-615 /-730 \mathrm{mV}$ in DMSO, which could not mediate the electron transfer in the NAD-GDH system. Thus, a mixed solution of two mediators in a 1:1 (Fe-PhenTPy: $\left.\mathrm{Fe}(\mathrm{CN})_{6}{ }^{3-}\right)$ molar ratio was prepared in DMSO containing 0.1 M TBAP. In this case, a pair of redox peaks of mixed mediators at a potential of $-51.8 /-157.6 \mathrm{mV}$ was observed (Fig. 1(C)), showing a negative shift of approximately $365 \mathrm{mV}$ (formal potential, $\Delta E_{\mathrm{p}}$ ) compared with Fe-PhenTPy. When the redox potentials of two species closely located or partly overlapped, their redox process can be interfered to each other, so that the two redox peaks are merged in a peak at the middle position of two redox potentials. In addition, to evaluate the electron-transfer rate of the mediators (Fe-PhenTPy, $\mathrm{Fe}(\mathrm{CN})_{6}{ }^{3-}$, and mixed mediators), the Nicholson method was applied. The electron-transfer rate constants of Fe-PhenTPy and $\mathrm{Fe}(\mathrm{CN})_{6}{ }^{3-}$, $k^{0}$, were calculated as $7.3 \times 10^{-4}$ and $1.7 \times 10^{-2} \mathrm{~cm} \mathrm{~s}^{-1}$, respectively. In the case of the mixture of Fe-PhenTPy and $\mathrm{Fe}(\mathrm{CN})_{6}{ }^{3-}, k^{0}$ was calculated to be $4.9 \times$ $10^{-3} \mathrm{~cm} \mathrm{~s}^{-1}$, which shows a 6.7-times enhanced value compared with that of Fe-PhenTPy. The results showed that it successfully tuned the redox potential of the mediator for the glucose sensor to reduce the interferences.

\section{Potential tuning of mediators on the NAD-GDH sensor}

We observed the voltammetric behavior of mediators adsorbed on the carbon electrode in an aqueous glucose solution to evaluate their performance for the NAD-GDH glucose sensor by measuring the oxidation potentials of Fe-PhenTPy with and without $\mathrm{Fe}(\mathrm{CN})_{6}{ }^{3-}$. Figure 2(A) shows the $\mathrm{CV}$ s recorded for the
Fe-PhenTPy modified electrode in a $200 \mathrm{mg} \mathrm{dL}^{-1}$ glucose solution. The onset potential $\left(E_{\text {onset }}\right)$, the anodic peak potential $\left(E_{\mathrm{pa}}\right)$, and anodic peak current $\left(\Delta I_{\mathrm{pa}}\right)$ of the Fe-PhenTPy coatedglucose sensor are $269.9,476 \mathrm{mV}$, and $9.7 \mu \mathrm{A}$, respectively. Mediators need to have a smaller oxidation potential than around $400 \mathrm{mV}$ in a glucose solution to reduce the interferences from oxidizing species in the present system; however, the Fe-PhenTPy-based glucose sensor has a slightly high oxidation potential. Thus, in order to tune the potential of the Fe-PhenTPy, we mixed the $\mathrm{Fe}(\mathrm{CN})_{6}{ }^{3-}$ in different molar ratios on the Fe-PhenTPy modified electrode. As the concentration of $\mathrm{Fe}(\mathrm{CN})_{6}{ }^{3-}$ increased from 0.5 to $5 \mathrm{mM}$, the oxidation potential shifted towards the negative direction, as shown in Fig. 2(B). The maximum potential tuning occurred using $5 \mathrm{mM} \mathrm{Fe}(\mathrm{CN})_{6}{ }^{3-}$, whereas the oxidation potential did not change more when the concentration of $\mathrm{Fe}(\mathrm{CN})_{6}{ }^{3-}$ increased to above $5 \mathrm{mM}$. In the case of a mixed mediator using $5 \mathrm{mM} \mathrm{Fe}(\mathrm{CN})_{6}^{3-}, E_{\text {onset }}, E_{\mathrm{pa}}$, and $\Delta I_{\mathrm{pa}}$ was $110 \mathrm{mV}, 280 \mathrm{mV}$, and $10.3 \mu \mathrm{A}$, respectively. Compared to Fe-PhenTPy, the oxidation potential was negatively shifted by approximately $200 \mathrm{mV}$ in an aqueous glucose solution. From this result, the oxidation potential of the mediator could be matched to the NAD-GDH system by simple mixing of the mediators.

Electrochemical impedance spectroscopy (EIS) was conducted to evaluate the charge-transfer resistance of each modified probe in a $0.1 \mathrm{M}$ PBS ( $\mathrm{pH} 7.4$ ) solution. Figure 3(A) shows Nyquist plots obtained for each layer of the bare, $\mathrm{Fe}-\mathrm{PhenTPy}, \mathrm{Fe}(\mathrm{CN})_{6}{ }^{3-}$ Fe-PhenTPy, and NAD-GDH/ $\mathrm{Fe}(\mathrm{CN})_{6}{ }^{3-} / \mathrm{Fe}-\mathrm{PhenTPy}$ modified on the SPCE. The charge-transfer resistance (Rp) was determined by fitting using Zview 2 impedance software. The $\mathrm{Rp}$ values of bare and Fe-PhenTPy were 695.4 and $250.8 \mathrm{~K} \Omega$, 

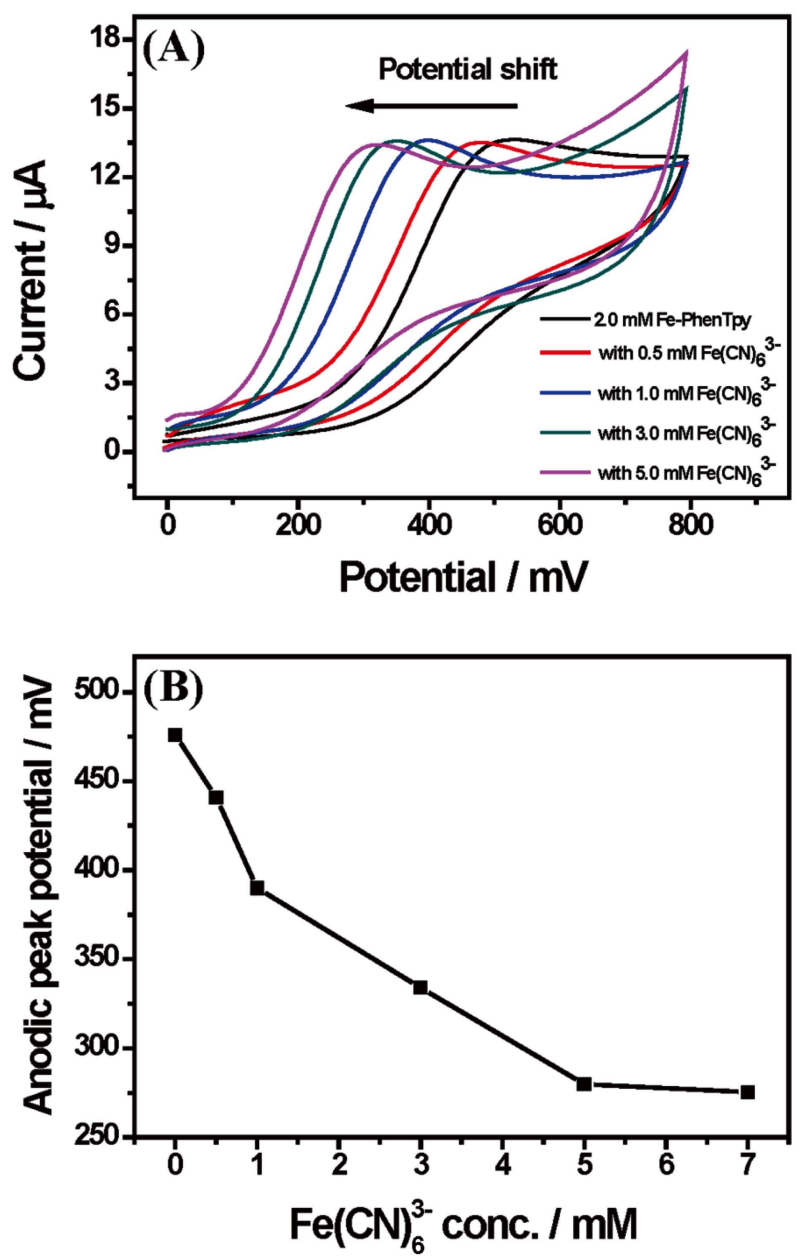

Fig. 2 (A) CVs recorded for glucose sensing with different ratios of $\mathrm{Fe}(\mathrm{CN})_{6}{ }^{3-}$ on the Fe-PhenTPy modified electrode in a $200 \mathrm{mg} \mathrm{dL}^{-1}$ glucose solution. (B) Effect of the potential tuning with $\mathrm{Fe}(\mathrm{CN})_{6}{ }^{3-}$ in different molar ratios.

respectively, indicating the Fe-PhenTPy adsorption onto the SPCE made the resistance lower of 2.8 times. The $5 \mathrm{mM}$ $\mathrm{Fe}(\mathrm{CN})_{6}{ }^{3-}$ mixed Fe-PhenTPy made the $R_{\mathrm{ct}}$ value lower to $109.8 \mathrm{~K} \Omega$, where it was decreased by 2.3 times compared to that of Fe-PhenTPy modified SPCE. The final NAD-GDH immobilized sensor probe coated with a mixed mediator shows an $R_{\text {ct }}$ value of $413.5 \mathrm{~K} \Omega$. In addition, the charge-transfer resistance was compared for the mediators mixed with $\mathrm{Fe}(\mathrm{CN})_{6}{ }^{3-}$ in different molar ratios on the Fe-PhenTPy modified electrode in Fig. 3(B). The $R_{\mathrm{p}}$ values of $\mathrm{Fe}(\mathrm{CN})_{6}^{3-} / \mathrm{Fe}-\mathrm{PhenTPy}$ were $232.3 \mathrm{~K} \Omega\left(0.5 \mathrm{mM} \mathrm{Fe}(\mathrm{CN})_{6}{ }^{3-}\right), 200.9 \mathrm{~K} \Omega\left(1.0 \mathrm{mM} \mathrm{Fe}(\mathrm{CN})_{6}{ }^{3-}\right)$, $138.6 \mathrm{~K} \Omega\left(3.0 \mathrm{mM} \mathrm{Fe}(\mathrm{CN})_{6}{ }^{3-}\right), 110.3 \mathrm{~K} \Omega\left(5.0 \mathrm{mM} \mathrm{Fe}(\mathrm{CN})_{6}{ }^{3-}\right)$, where the $R_{\mathrm{p}}$ value decreased as the concentration of $\mathrm{Fe}(\mathrm{CN})_{6}{ }^{3-}$ increased. This result indicates that the mixed mediators cause the sensor probe to be more conductive, and to facilitate the electron transfer process. Thus, the optimized concentration of $\mathrm{Fe}(\mathrm{CN})_{6}{ }^{3-}$ was $5.0 \mathrm{mM}$ and coated on the Fe-PhenTPy mediator modified probe.

\section{Interference effects}

The selectivity of the NAD-GDH/Fe $(\mathrm{CN})_{6}{ }^{3-} / \mathrm{Fe}-\mathrm{Ph}$-nTPy sensor strip was evaluated with human blood samples in the presence of an exogenous interfering species, such as acetaminophen, ascorbic acid, bilirubin, cholesterol, creatinine, dopamine, EDTA, galactose, and uric acid, etc. According to
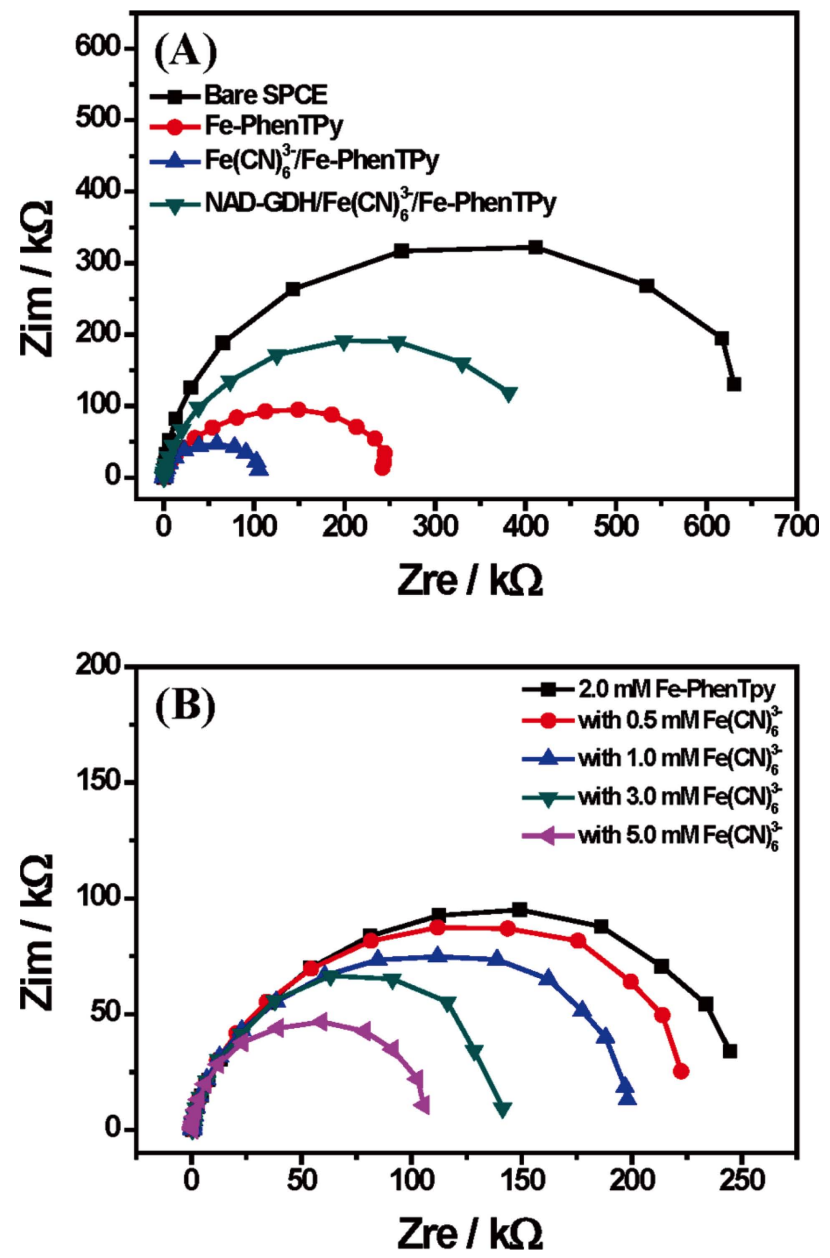

Fig. 3 Nyquist plots of impedance measurements for (A) bare SPCE, Fe-PhenTPy, Fe-PhenTPy/Fe $(\mathrm{CN})_{6}^{3-}$, and NAD-GDH/FePhenTPy $/ \mathrm{Fe}(\mathrm{CN})_{6}{ }^{3-}$, (B) $\mathrm{Fe}(\mathrm{CN})_{6}{ }^{3-}$ coated on the Fe-PhenTPy in different molar ratios.

Table 1 List of potential exogenous interfering substances concentration

\begin{tabular}{lclc}
\hline $\begin{array}{c}\text { Interfering } \\
\text { substance }\end{array}$ & $\begin{array}{c}\text { Spike } \\
\text { concentration/ } \\
\text { mg dL }^{-1}\end{array}$ & $\begin{array}{c}\text { Interfering } \\
\text { substance }\end{array}$ & $\begin{array}{c}\text { Spike } \\
\text { concentration/ } \\
\text { mg dL }^{-1}\end{array}$ \\
\hline Acetaminophen & 4 & Maltose & 200 \\
Ascorbic acid & 3 & Methyldopa & 2.5 \\
Bilirubin & 20 & Salicylate & 60 \\
Cholesterol & 500 & Tolazamide & 5 \\
Creatinine & 30 & Uric acid & 23.5 \\
Dopamine & 13 & Xylose & 15 \\
EDTA & 720 & Heparin & 2.8 \\
Galactose & 45 & Ibuprofen & 50 \\
Gentisic acid & 7.5 & Tolbutamide & 100 \\
Hemoglobin & 200 & Triglyceride & 3000 \\
L-DOPA & 5 & & \\
\hline
\end{tabular}

the ISO 15197 criteria, the above-mentioned interfering substances should have less than $10 \%$ of glucose response in a blood sample. ${ }^{13}$ Some interferences, such as acetaminophen, ascorbic acid, and dopamine, were sometimes serious, which possess a similar oxidation potential to that of glucose. Thus, we tried to reduce any interferences by oxidation potential 


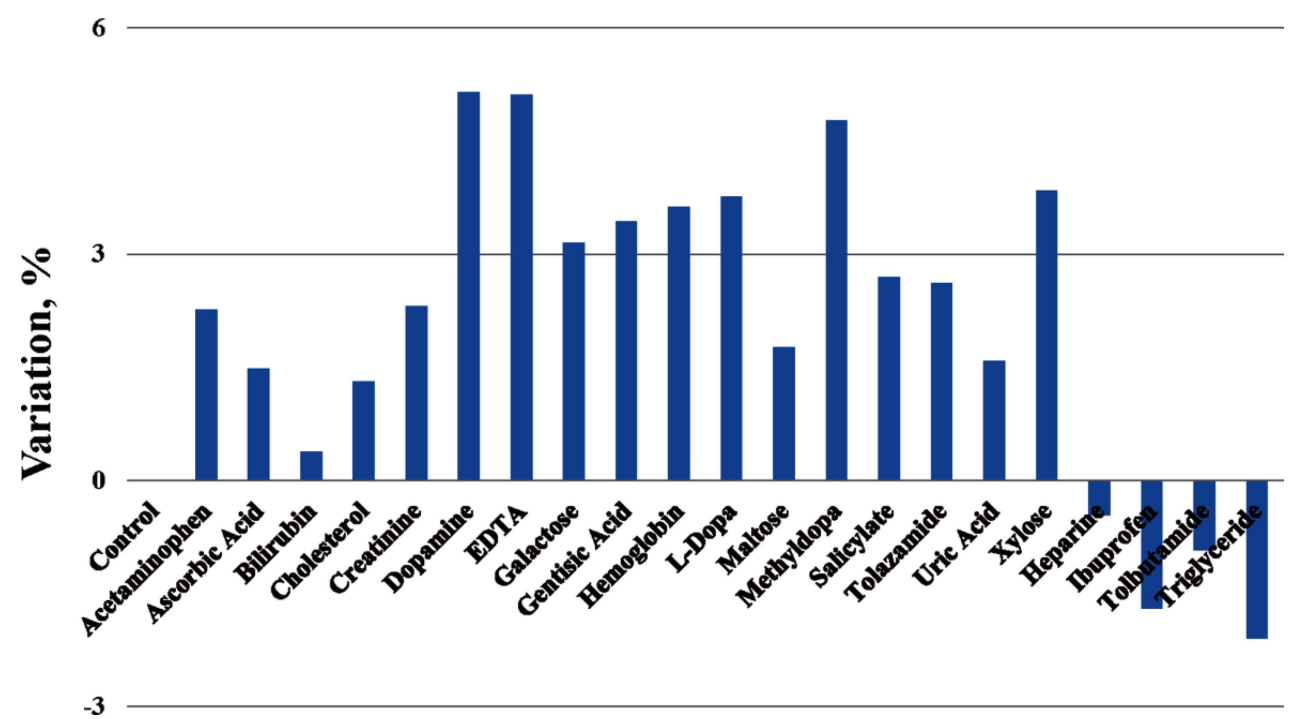

Fig. 4 Variation values of the exogenous interferences in human blood.

tuning of the mediator adsorbed on the glucose sensor strip. All interfering test samples were then spiked with a different concentration of the respective interfering substance to the control sample (Table 1). In this case, the glucose level of the human blood as a control sample was adjusted to $120 \mathrm{mg} \mathrm{dL}^{-1}$. Figure 4 shows the variation of the amperometric response for control and test samples at an applied potential of $350 \mathrm{mV}$. Although the variation for test samples was the maximum with $5 \%$ higher than that of the control sample, the value is within the measuring error range of $10 \%$. The effect of exogenous materials in human blood can be minimized by modulating the oxidation potential of the mediator involved in enzymatic glucose oxidation below $400 \mathrm{mV}$.

\section{Evaluation of glucose sensing performance}

$\mathrm{CV}$ s recorded for the sensor probe shows an oxidation peak of the modified mediator at $280 \mathrm{mV}$. Based on this value, a potential of $350 \mathrm{mV}$ was applied for $5 \mathrm{~s}$ in the chronoamperometric analysis. As shown in Fig. 5, the calibration curve for glucose detection was obtained at different concentrations of glucose in a 0.1 M PBS ( $\mathrm{pH} 7.4)$ with the home-made glucose meter. The measured currents $(\Delta I)$ for various glucose concentrations were obtained from the baseline signal at $5 \mathrm{~s}$. The proposed sensor exhibits a dynamic range of between 30 to $500 \mathrm{mg} \mathrm{dL}^{-1}$ with a good sensitivity of $0.57 \mu \mathrm{A} \mathrm{mM}^{-1}$. The linear dependence yielded a regression equation of $\Delta I(\mu \mathrm{A})=(0.124 \pm 0.111)+(0.032 \pm 0.001) \quad[\mathrm{C}]$ $\left(\mathrm{mg} \mathrm{dL}^{-1}\right)$ with a correlation coefficient of 0.997 . The detection limit was determined to be $14.81 \mathrm{mg} \mathrm{dL}^{-1}$ from blank noise signals (95\% confidence level, $k=3, n=10$ ). The reproducibility of the data was examined using ten different sensors that reveal a relative standard deviation of $2.5 \%$ at a glucose concentration of $200 \mathrm{mg} \mathrm{dL}^{-1}$.

\section{Glucose meter fabrication}

A block diagram of the amperometric glucose meter is shown in Fig. 6(A). The meter consists of potential control amplifier, feedback amplifier, current follower (IV converter), low-pass filter, and micro control unit (MCU). Potential control and feedback amplifier were used to maintain the potential between working and reference electrodes. The response current in proportion to the concentration of glucose is converted to a

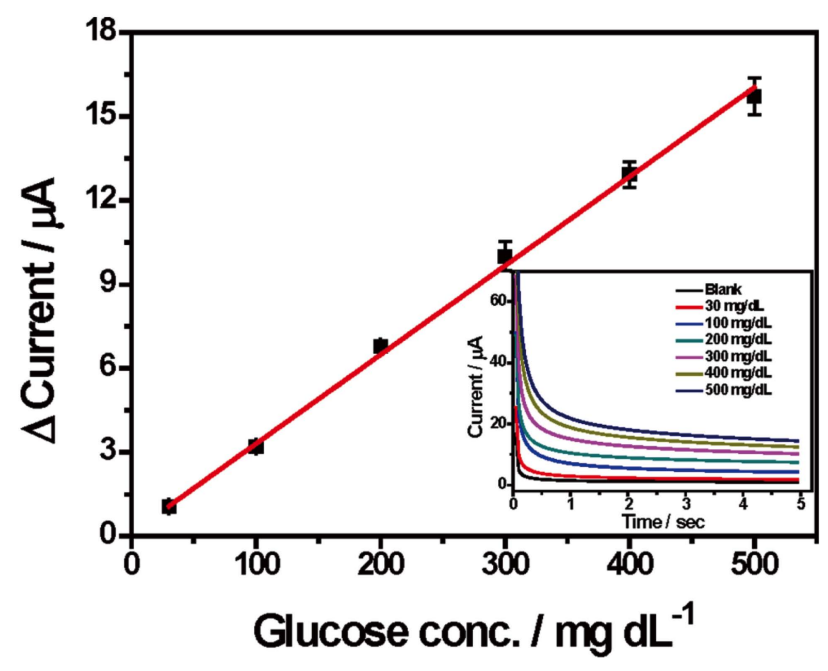

Fig. 5 Linear calibration plots for various glucose concentrations from 30 to $500 \mathrm{mg} \mathrm{dL}^{-1}$ using amperometric method. The applied potential was set at $350 \mathrm{mV}$. Inset: amperometric responses recorded for different glucose concentration.

potential by a current follower. A low-pass filter provided a smoother signal by removing the noise signal, and the thenobtained signal was transferred to a 12-bit ADC (analogy to digital converter) of $\mathrm{MCU}$ for further processing the digital signal. In addition, we made-up a cartridge type product including multiple sensor strips accommodated in one case in order to satisfy the user's convenience. The portable electrochemical glucose meter was equipped with a dozen sensor strips, which has to be replaced each time efficiently.

In addition, the alignment of the electrodes in the sensor strip was also tried, because it is crucial to reveal the effective response of the sensor to target molecules. Since the counter electrode in the conventional biosensor faces only one surface with the working electrode, it makes a too-small effective area to derive a sufficient amount of a reaction between them. Thus, we modified the alignment of the sensor structures to give an enhanced response. Here, the counter electrode of the sensor 


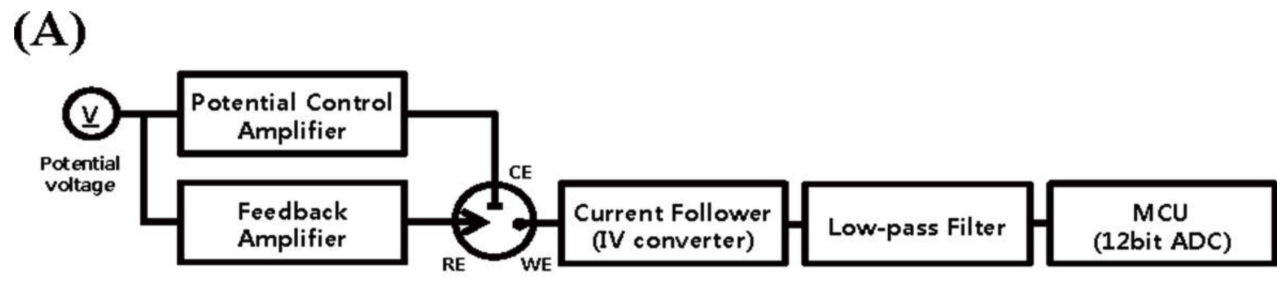

(B)

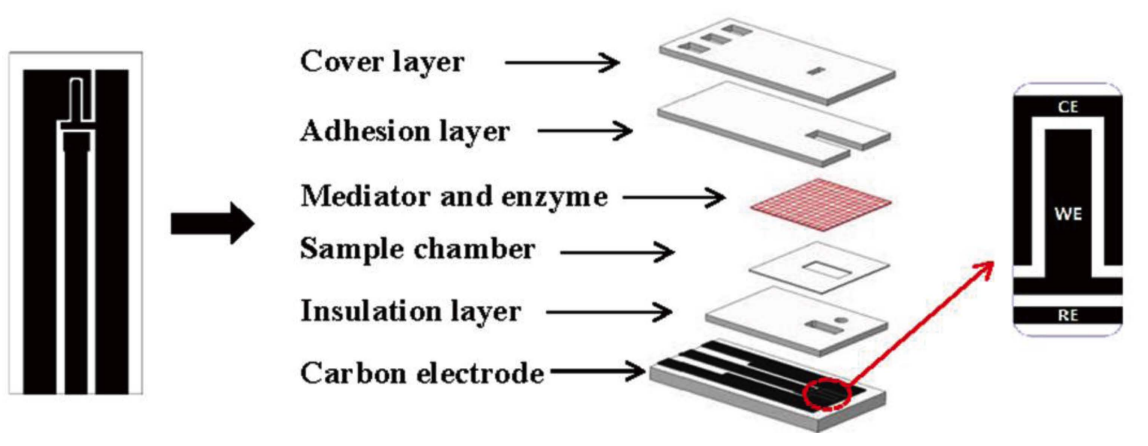

Fig. 6 (A) Block diagram of the glucose meter circuit. (B) Schematic diagram of the glucose sensor strip.

surrounded the whole peripheral surfaces of the working electrode and facing much electrode area (Fig. 6(B)). This alignment proved to be 4.7 -fold more sensitive than that of the conventional electrode alignment, although the working electrode area increased by 1.5 times in this structure.

\section{Conclusions}

Although Fe-PhenTPy exhibited an excellent performance for an NAD-GDH glucose sensor, it has a slightly high oxidation potential of $476 \mathrm{mV}$, and it responds to the interferences from easily oxidizing species present in blood. To avoid any exogenous interfering species, we tried to reduce the oxidation potential of Fe-PhenTPy by mixing with $\mathrm{Fe}(\mathrm{CN})_{6}{ }^{3-}$. The oxidation potential of the mediator was successfully tuned to be $280 \mathrm{mV}$ for the NAD-GDH glucose sensor. Therefore, exogenous substances do not interfere with the detection of glucose as is within the error range 5\%. In addition, a new cartridge type glucose meter was developed that included a multiple glucose sensor strip so as to enhance the user convenience as well as the alignment of the electrodes structure, which showed an enhanced sensitivity compared with that of conventional sensors.

\section{Acknowledgements}

This work was supported by the National Research Foundation of Korea (NRF) grant funded by the Korea government (MISP) (Grant No. 2010-002-9128), and Healthcare \& Sensing Lab. Advanced Device Team, DMC R\&D center of Samsung
Electronics Co., Ltd. (Suwon, S. Korea).

\section{References}

1. M. Brownlee, Nature, 2001, 414, 813 .

2. L. Clarke, D. Cox, L. Goder-Frederick, W. Carter, and S. Pohl, Diabetes Care, 1987, 10, 622.

3. D. Nakayama, Y. Takeoka, M. Watanabe, and K. Kataoka, Angew. Chem., 2003, 115, 4329.

4. J. C. Pickup, F. Hussain, N. D. Evans, O. J. Rolinski, and D. J. S. Birch, Biosens. Bioelectron., 2005, 20, 2555.

5. S. K. Mahadeva and J. Kim, Sens. Actuators, B, 2011, 157, 177.

6. J. Wang, Chem. Rev., 2008, 108, 814 .

7. L. Gorton and E. Dominguez, Rev. Mol. Biotechhnol., 2002, 82, 371.

8. Y. Yamashita, S. Ferri, M. L. Huynh, H. Shimizu, H. Yamaoka, and K. Sode, Enzyme Microb. Technol., 2013, 52, 123.

9. M. Zayats, E. Katz, R. Baron, and I. Willner, J. Am. Chem. Soc., 2005, 127, 12400.

10. J. P. Frias, J. M. Ellison, C. G. Lim, and C. M. Montandon, Diabetes Care, 2010, 33, 728.

11. N. J. Forrow, G. S. Sanghera, J. L. Watkin, and S. Walters, Biosens. Bioelecton., 2005, 20, 1617.

12. D.-M. Kim, M. Y. Kim, S. S. Reddy, J. Cho, C. H. Cho, S. Jung, and Y.-B. Shim, Anal. Chem., 2013, 85, 11643.

13. International Organization for Standardization, "In vivo diagnostic test system-Requirements for blood-glucose monitoring systems for self-testing in managing diabetes mellitus", ISO 15197, 2013. 